\title{
Formulation, Characterization and In vitro Evaluation of Capecitabine Loaded Polycaprolactone-Chitosan Nanospheres
}

\author{
Prakash Katakam $^{1}$, Yadagiri Phalguna ${ }^{2,3}$ and Dommati Harinarayana ${ }^{4}$ \\ ${ }^{1}$ Faculty of Pharmacy, University of Zawia, Al-Zawia, Libya \\ ${ }^{2}$ Department of Pharmaceutics, Jyothismathi college of Pharmacy, Thurkapally (V) \\ Shameerpet (M), R.R. Dist-500078 (A.P)-India \\ ${ }^{3}$ Jawaharlal Nehru Technological University, Hyderabad \\ ${ }^{4}$ Nishka Laboratories, Madhavi Apartment, Uppal, Hyderabad-500039, India
}

\begin{abstract}
The aim of this study is to formulate the capecitabine loaded nanospheres of polycaprolactone-chitosan, cross linked with Tripolyphosphate for anticancer therapy, in order to enhance the bioavailability and to reduce the dose frequency. Formulations of capecitabine loaded nanospheres were prepared by double emulsion solvent evaporation and solvent diffusion methods. Fourier transmission infrared spectroscopy studies indicated no chemical interaction between drug and polymer. Scanning electron microscopy showed nanospheres having a discrete spherial structure without aggregation. The average particle size was found as $616 \pm 110$ to $713 \pm 115 \mathrm{~nm}$. In vitro release studies were performed by the dialysis membrane method. All the drug loaded batches were follwed zero order and sustained drug release over a period of $24 \mathrm{hrs}$.
\end{abstract}

Key words: Capecitabine, Nanospheres, Double emulsion solvent Evaporation, Solvent diffusion.

\section{Introduction}

The major goals in designing nanoparticles as a delivery system is to control particle size, surface properties and release of pharmacologically active agents in order to achieve the site-specific action of the drug at the therapeutically optimal rate and dose regimen $(\mathrm{Mu}$ et al., 2003). Nanoparticles are defined as particulate dispersions or solid particles with a size in the range of 10$1000 \mathrm{~nm}$. The drug is dissolved, entrapped, encapsulated or attached to a nanoparticle matrix. Depending upon the method of preparation, nanoparticle, nanospheres or nanocapsules can be obtained. Nanospheres are matrix systems in which the drug is physically and uniformly dispersed. Capecitabine is a pro-drug that is converted to fluorouracil in the body tissues following the oral administration. It is widely used in the treatment of metastatic colorectal cancer and breast cancer, since it is readily absorbed from the gastrointestinal tract. The adverse effects associated with capecitabine include bonemarrow depression, cardiotoxicity, diarrhoea, nausea and vomiting, stomatitis, dermatitis etc. Besnard et al., (2008) studied on optimization of blood sampling with cytidine deaminase inhibitor for improved analysis of capecitabine metabolites in biological fluids. Wong HL et al., (2007) studied passive targeting of capecitabine nanoparticles in tumor bearing rats. The PEGylated and PLGA nanoparticles by a single emulsion method in which drug encapsulation efficiency is not compromised (Jason et al., 2009). Paclitaxel loaded PCL/PEG nanoparticles were prepared by single emulsion technique in which $100 \mathrm{~nm}$ particle size is obtained with a release of $72.5 \pm 2.4 \%$ (Nazneen et al., 2012). Intravesical cationic nanoparticles of chitosan and PCL for delivery of mitomycin C has been studied (Erem Bilonsy et al., 2009). Nanoparticles diameter varied from 180 to $300 \mathrm{~nm}$ with 2 to 3 fold increase of encapsulation efficiency.

In the present study nanospheres was developed by using PCL and Chitosan as carriers. The objective of the study was to formulate the nanospheres by two different methods such as double emulsion solvent evaporation method and solvent diffusion method as well as to investigate the release profiles of capecitabine nanospheres. 


\section{Materials and Methods}

Capecitabine was a gift sample from the Dr. Reddy's Lab., Hyderabad, India. Polycaprolactone (Mol. wt 14,000), Chitosan (Mol. wt. 60,000-120,000), Tripolyphosphate (Mol. wt.367.86), Polyvinyl alcohol (Mol. wt.7, 200) were purchased from Sigma Aldrich Pvt. Ltd, Bangalore, India and Dialysis membrane (LA-395-5Mt) from Himedia Pvt. Ltd, Mumbai, India. All other reagents were of analytical grade.

Capecitabine loaded nanospheres were formulated using: Polycaprolactone, Chitosan, Tripolyphosphate, Polyvinylalcohol combinations at various concentrations. Formulations were prepared by double emulsion solvent evaporation method (CPN1-CPN3) and solvent diffusion method (CPN4-CPN6) each using polycaprolactone (0.5, 1.5 and 2.5\%), Chitosan (0.5, 1.5 and 2.5\%), polyvinylalcohol (4\%), tripolyphosphate (1\%). All ingredients were calculated on the basis of $\% \mathrm{w} / \mathrm{w}$.

Double emulsion $\left(W_{1} / O / W_{2}\right)$-solvent evaporation method: In this method the internal aqueous phase (IAP) was prepared by taking $20 \mathrm{ml}$ of double distilled water and OP (organic phase) was prepared by dissolving the capecitabine $150 \mathrm{mg}$, PCL (Mochizuki et al.,1997) in 15 $\mathrm{ml}$ of DCM (dichloromethane) and chitosan in $5 \mathrm{ml}$ of acetic acid (Lifeng et al., 2005) was taken. The IAP (internal aqueous phase) was added slowly drop by drop into the organic phase by continuously stirring at 2000 rpm in a magnetic stirrer (2MLH, Remi Equipment Pvt. Ltd., India) to make a primary emulsion. For the formation of emulsion $20 \mathrm{ml}$ of external acquired phage EAP, containg 4\% PVA was added drop wise into the primary emulsion during sonication. Then $3 \mathrm{ml}$ of TPP $(1 \% \mathrm{~W} / \mathrm{W})$ was added drop wise into the above solution. Then the secondary emulsion was kept in magnetic stirrer at 800 rpm for overnight for the excess DCM to evaporate. Particles were separated through centrifugation at 15000 rpm (Remi Equipment, RM-12 Microcentrifuge, Pvt. Ltd. Mumbai, India) for 20 mins (Lee et al., 2006).

Solvent diffusion method: In solvent diffusion method the organic solution was prepared by dissolving the polymers PCL in $6 \mathrm{ml}$ of dichloromethane and chitosan in $5 \mathrm{ml}$ of acetic acid. The capecitabine $150 \mathrm{mg}$ was dissolved in $5 \mathrm{ml}$ of DCM and was poured drop by drop into the above organic solution. The polymeric drug solution obtained was then added into the aqueous PVA solution $(4 \%, \mathrm{w} / \mathrm{w})$ in a $100 \mathrm{ml}$ glass beaker. Then TPP $(1 \% \mathrm{w} / \mathrm{w})$ was mixed continuously by stirring at $800 \mathrm{rpm}$ with a Magnetic stirrer for $4 \mathrm{hrs}$ until the emulsion was formed (Leroux et al., 1995). During the continuation of mixing the dispersion was condensed by adding double distilled water up to $100 \mathrm{ml}$. This condensation process was repeated three times to remove the residual PVA and organic solvents (Quintanar et al., 1998). Particles were separated through centrifugation at $15000 \mathrm{rpm}$.

Fourier transform infra-red (FT-IR) spectroscopy analysis: IR spectral analysis of pure drug capecitabine and polymers was carried out and observation was made whether changes occurred in chemical constitution of drug after combining it with the polymers. The samples were crushed with $\mathrm{KBr}$ to get pellets by applying pressure on $600 \mathrm{Kg} / \mathrm{cm}^{2}$ and scanned with the IR instrument (Shimadzu, 8400 Series, Tokyo, Japan) from 400-4000 $\mathrm{cm}^{-1}$ (Zhang et al., 2003).

DSC studies: Thermo grams were obtained by using (Netzsch Tech. DSC 200F3, Selb, Germany) at a heating rate $10^{\circ} \mathrm{C} / \mathrm{min}$ over a temperature range of $35-250^{\circ} \mathrm{C}$. The sample was hermetically sealed in an aluminium crucible. Nitrogen gas was purged at a rate of $10 \mathrm{ml} / \mathrm{min}$ for maintaining inert atmosphere (Rahul et al., 2011).

Estimation of amount of drug incorporated to PCLchitosan nanospheres: Accurately weighed $100 \mathrm{mg}$ of nanospheres were powdered in a mortar and suspended in $100 \mathrm{ml}$ of phosphate buffer (pH 7.4) and kept in sonication for $2 \mathrm{hrs}$. Then the samples were centrifuged at 1000rpm for 20mins to remove the supernatant layer, if any. The samples were filtered. From this filtered solution $1 \mathrm{ml}$ of sample was withdrawn and diluted to $25 \mathrm{ml}$ with phosphate buffer (pH 7.4). Then it was analyzed spectrophotometrically at $240 \mathrm{~nm}$.

Zeta potential analysis: The zeta potential was measured using the appropriate instrument (Beckman Coulter Delsa Nano C, Brea, USA). The sample was diluted with double distilled water and taken in the cuvettes and temperature maintained at $25^{\circ} \mathrm{C}$ (Sandri et al., 2010).

Particle size analysis: The size distribution of the nanospheres was determined using the particle size analyzer (Beckman Coulter, Delsa nano C, Brea, USA) equipped with a dry accessory system. Sample was diluted 
with water and temperature maintained at $25^{\circ} \mathrm{C}$ (Donbrow et al., 1980).

Scanning electron microscopy: Scanning electron microscopy (JEOL 5400, Tokyo, Japan) was used to determine the shape, surface topography and texture as well as to examine the morphology of fractured or sectioned surface. SEM is a commonly used method for characterizing drug delivery systems, owing in large part to simplicity of sample preparation and ease of operation. Sample spreads on the small square plate and coated with a gold ion for 5-6 mins. The prepared sample was kept inside the chamber and images captured with different magnifications.

In vitro drug release studies: In vitro release of Capecitabine nanospheres was conducted by a dialysis membrane having pore size of $2.4 \mathrm{~mm}$ (LA-395-5Mt Himedia Pvt. Ltd, Mumbai, India) with $75 \mathrm{ml}$ of $\mathrm{pH} 7.4$ phosphate buffer at $37^{\circ} \mathrm{C}$. Briefly in a $100 \mathrm{ml}$ beaker 75 $\mathrm{ml}$ of $\mathrm{pH} 7.4$ phosphate buffer was taken. A $2 \mathrm{ml}$ of formulation was taken into a dialysis bag and dipped into the buffer solution. The dialysis membrane was activated prior using by soaking in $1 \% \mathrm{w} / \mathrm{v} \mathrm{NaOH}$ over night. The flask was kept on a magnetic stirrer. Stirring was maintained at $250 \mathrm{rpm}$ and the temperature of the buffer was maintained at $37^{\circ} \mathrm{C}$. Sampling was done by withdrawing $5 \mathrm{ml}$ of aliquots from a beaker. Immediately $5 \mathrm{~mL}$ of fresh buffer was added to maintain the sink condition. Samples were analyzed after adequately diluting with methanol by using a UV-Visible Spectrophotometer (UV/VIS-Double beam Spectrophotometer, V-530, Jasco, Tokyo, Japan) at a wave length of $240 \mathrm{~nm}$.

Drug release kinetics (Higuchi et al., 1961): In order to investigate the mode of release from the nanospheres, the release data were fitted into zero-order, first-order, Higuchi, Korsmeyer-Peppas equations (Ritger et al., 1987). The regression equations were calculated and the correlation coefficients were determined.

Stability studies: The stability studies of optimized formulations were stored in the high density polyethylene (HDPE) containers, tightly closed and kept at $45 \pm 2{ }^{\circ} \mathrm{C}$ and $75 \pm 5 \%$ relative humidity for 90 days. The samples were characterized for change in various parameters such as particle size, zetapotential, drug content estimation.

\section{Results and Discussion}

Capecitabine nanospheres were successfully prepared by the double emulsion solvent evaporation method and solvent diffusion method. Nanospheres were examined for various evaluation parameters. The drug entrapment for CPN3 showed in the range of $85.46 \pm 0.25$ and in CPN6 $96.53 \pm 0.86$. Particle size of nanospheres was found to be $616 \pm 110$ to $713 \pm 115 \mathrm{~nm}$. The characteristics of the prepared nanospheres are summarized in (Table 1).

Table 1. Drug loading and Entrapment efficiency of prepared nanospheres.

\begin{tabular}{lcc}
\hline Formulation & $\begin{array}{c}\text { Drug loading } \\
(\%)\end{array}$ & $\begin{array}{c}(\%) \text { Drug } \\
\text { entrapment }\end{array}$ \\
\hline CPN1 & $71.05 \pm 0.06$ & $74.82 \pm 0.14$ \\
CPN2 & $78.56 \pm 0.58$ & $80.24 \pm 0.10$ \\
CPN3 & $83.21 \pm 0.12$ & $85.46 \pm 0.25$ \\
CPN4 & $74.43 \pm 0.25$ & $88.29 \pm 0.43$ \\
CPN5 & $80.37 \pm 0.25$ & $92.41 \pm 0.35$ \\
CPN6 & $85.28 \pm 0.30$ & $96.53 \pm 0.86$ \\
\hline
\end{tabular}

FT-IR spectra acquired were taken for drug and polymer for the possible drug-excipient study. A broad strong characteristic peak at $3518.4 \mathrm{~cm}^{-1}$ corresponds to $\mathrm{N}-\mathrm{H}$ Stretching and $\mathrm{C}-\mathrm{H}$ stretching for the pure Capecitabine at $2961 \mathrm{~cm}^{-1}$ and characteristic peak at 1715 $\mathrm{cm}^{-1}$ corresponds to $\mathrm{C}=\mathrm{O}$ stretching. A peak at $1502.3 \mathrm{~cm}^{-1}$ represents aromatic $\mathrm{C}=\mathrm{C}$.

The carbon-oxygen bonds stretching in the region of $700 \mathrm{~cm}^{-1}$ to $1500 \mathrm{~cm}^{-1}$ is from PCL components, while the bands of carbonyl, $\mathrm{C}=\mathrm{O}$ at $1739.5 \mathrm{~cm}^{-1}$ and symmetric and asymmetric of $\mathrm{CH}_{2}$ at $2867 \mathrm{~cm}^{-1}$ and $2947.9 \mathrm{~cm}^{-1}$ separately are also from the PCL.

The FTIR spectrum of Chitosan showed a broad-OH stretching absorption band between 3600 and $3100 \mathrm{~cm}^{-1}$. Another major absorption band was seen in between 1220 and $900 \mathrm{~cm}^{-1}$, which represented the free amino group $\left(-\mathrm{NH}_{2}\right)$ at $\mathrm{C} 2$ position of Glucosamine, a major peak present.

Differential Scanning Calorimetry (DSC) of the drug and polymer was performed to determine the drugexcipient interaction. The DSC curve of chitosan showed an endothermic peak at $102.07^{\circ} \mathrm{C}$ and PCL showed an endothermic peak at $68^{\circ} \mathrm{C}$ which represented the melting process. The drug showed an endothermic peak at $123^{\circ} \mathrm{C}$ which represented the melting process. 
Scanning electron microscopic (SEM) photographs showed nanospheres of discrete nature, and distinct particle size and shape with a smooth surface.

Particle size analysis was performed on the optimized formulation; optimization was based upon the drug release study. Particle size analysis was performed in order to investigate whether the particle size is within the range (Table 2). The maximum diameter of the particles was found to be $616 \pm 110$ to $713 \pm 115 \mathrm{~nm}$ (Figure 2).
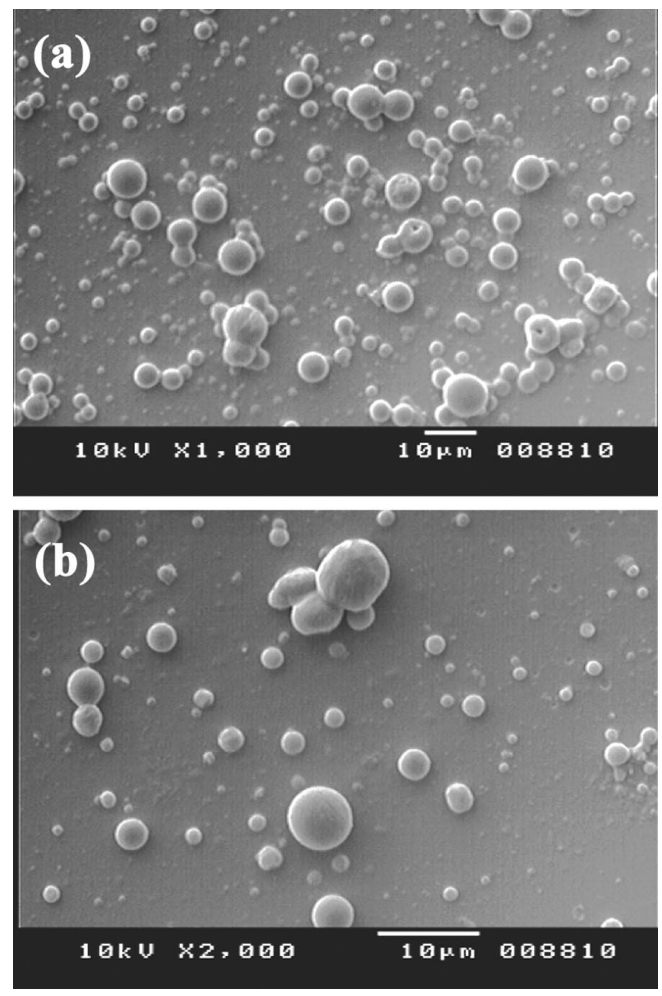

Figure 1 (a). SEM photograph of CPN 6. (b). SEM photograph of CPN 6.

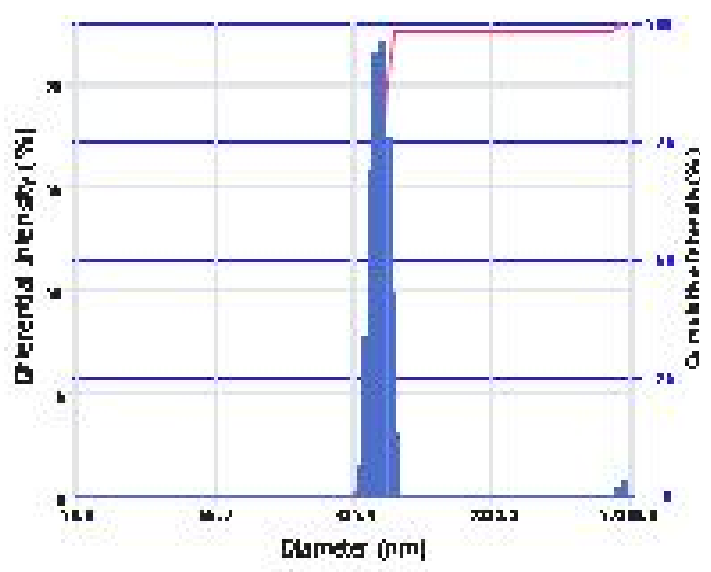

Figure 2. Particle size analysis of formulation CPN6.
Zeta potential was used to determine the electrophoretic mobility of particles. The magnitude of the zeta potential gives an indication of the potential stability of the colloidal system. The zeta potential was found to be $-17.30 \mathrm{mV}$ (Figure 3).



Figure 3. Zeta potential of formulation CPN 6.

Table 2. Particle size and Zeta potential Analysis of formulation CPN 6.

\begin{tabular}{|c|c|c|c|}
\hline \multicolumn{2}{|c|}{ Particle size analysis } & \multicolumn{2}{|c|}{ Zeta potential } \\
\hline Diameter & $\begin{array}{l}850.8 \\
(\mathrm{~nm})\end{array}$ & Zeta potential & $-17.30(\mathrm{mV})$ \\
\hline $\begin{array}{l}\text { Poly } \\
\text { dispersity } \\
\text { index }\end{array}$ & 0.316 & Mobility & $\begin{array}{l}-1.34 \\
\left(\mathrm{~cm}^{2} / \mathrm{Vs}\right)\end{array}$ \\
\hline $\begin{array}{l}\text { Diffusion } \\
\text { constant }\end{array}$ & $\begin{array}{l}5.76 \\
\left(\mathrm{~cm}^{2} / \mathrm{sec}\right)\end{array}$ & Conductivity & $\begin{array}{l}3.78 \\
(\mathrm{mS} / \mathrm{cm})\end{array}$ \\
\hline $\begin{array}{l}\text { Refractive } \\
\text { index }\end{array}$ & 1.33 & Refractive index & 1.33 \\
\hline Viscosity & $0.88(\mathrm{cP})$ & Viscosity & $0.887(\mathrm{cP})$ \\
\hline
\end{tabular}

The drug release of prepared nanospheres with different combination of polymers PCL and chitosan of different ratios showed slower release when there was an increase in the concentration. It was also observed that drug entrapment and drug loading also affect the drug release from nanospheres. The formulation CPN1 showed $98.43 \pm 0.52 \%$ release of the drug for a period of $24 \mathrm{hrs}$. The CPN 3 showed drug release of $95.32 \pm 0.45 \%$ for a period of 24hrs (Figure 4). When the drug release profiles are fitted to release kinetics the slope for Peppas was in the range of 0.45 to 1.00 indicating both diffusion and erosion of both biodegradable polymers.

The formulation CPN4 showed $98.84 \pm 0.311 \%$ release of the drug for a period of $24 \mathrm{hrs}$, while the CPN 6 


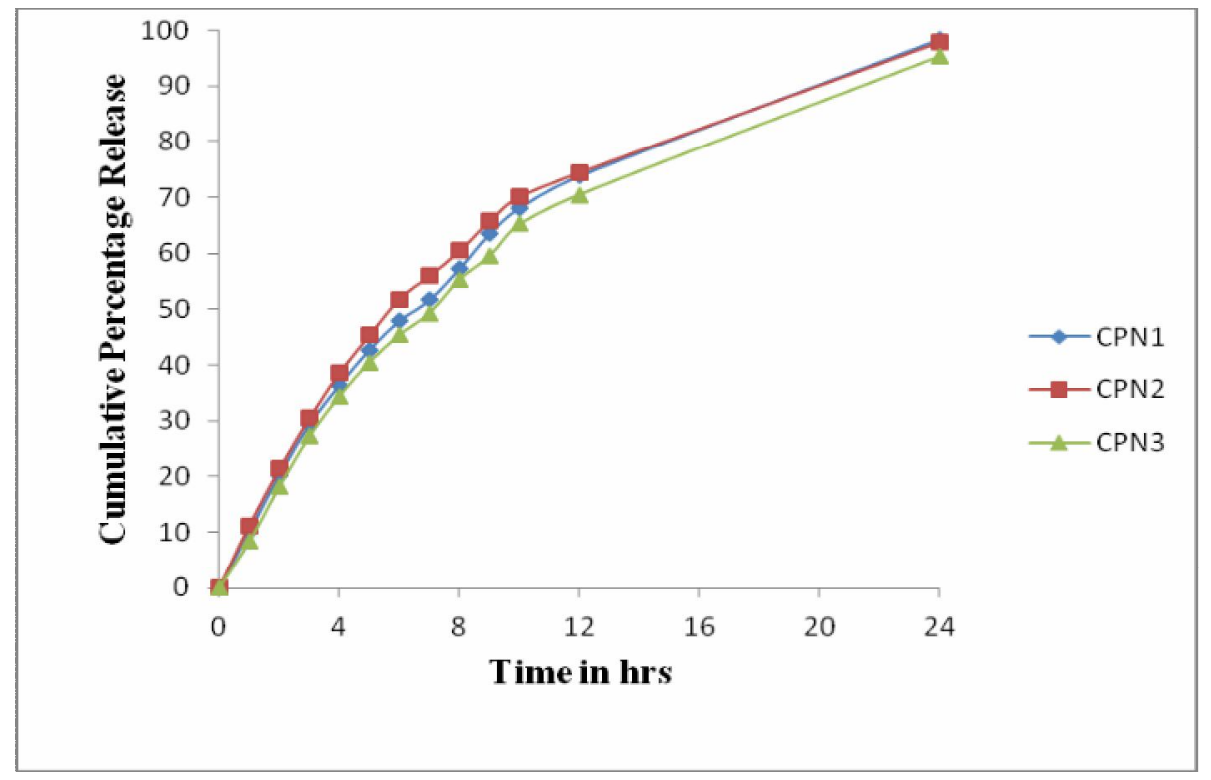

Figure 4. Cumulative percentage release vs time plots of CPN 1 to CPN 3.

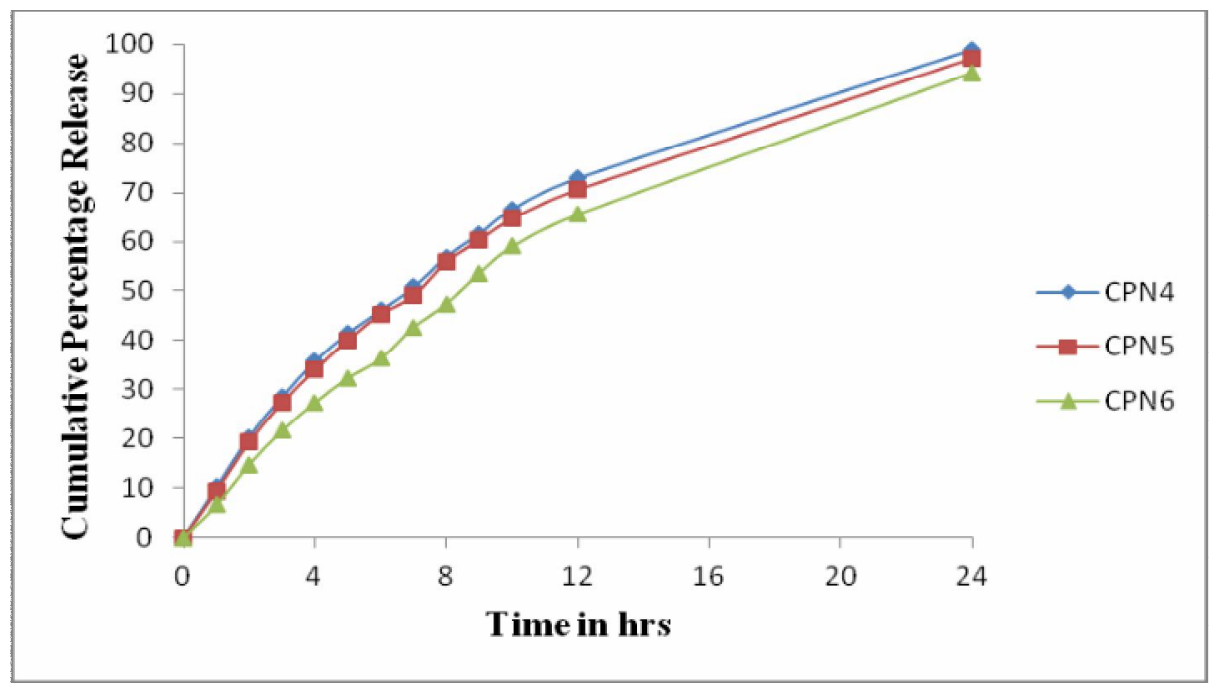

Figure 5. Cumulative percentage release vs time plots of CPN 4 to CPN 6.

Table 3. Kinetic studies of prepared nanospheres.

\begin{tabular}{lccccc}
\hline Formulation code & Zero order & First order & Higuchis & \multicolumn{2}{c}{ Peppas } \\
\cline { 2 - 6 } & $\mathrm{r}^{2}$ & $\mathrm{r}^{2}$ & $\mathrm{r}^{2}$ & $\mathrm{r}^{2}$ & $\mathrm{n}$ \\
\hline CPN1 & 0.857 & 0.949 & 0.985 & 0.973 & 0.725 \\
CPN2 & 0.884 & 0.955 & 0.977 & 0.968 & 0.697 \\
CPN3 & 0.887 & 0.984 & 0.987 & 0.964 & 0.770 \\
CPN4 & 0.902 & 0.929 & 0.990 & 0.978 & 0.721 \\
CPN5 & 0.900 & 0.962 & 0.991 & 0.976 & 0.740 \\
CPN6 & 0.934 & 0.970 & 0.994 & 0.982 & 0.844 \\
\hline
\end{tabular}

$\mathrm{r}^{2}$ corelation coefficient 
showed drug release of $94.28 \pm 0.20 \%$ for a period of 24hrs (Figure 5). When the drug release profiles are fitted to release kinetics the slope for Peppas was in the range of 0.45 to 1 (Table 3 ) indicating both diffusion and erosion of both biodegradable polymers.

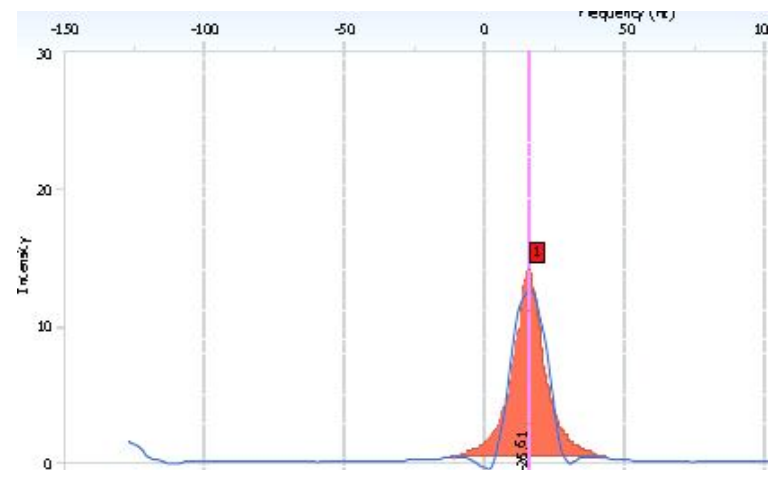

Figure 6. Particle Size analysis of Formulation CPN6

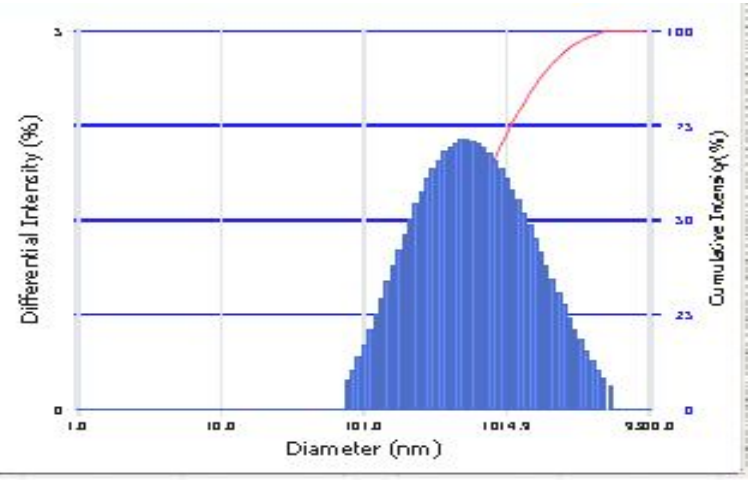

Figure 7. Zeta potential of Formulation CPN 6.

Table 4. Particle size and zetapotential analysis after stabilitystudies of formulationCPN 6.

\begin{tabular}{lll}
\hline Particle size analysis & Zetapotential & \\
\hline Diameter & Zetapotential & $-26.61(\mathrm{mV})$ \\
$\begin{array}{l}890.9(\mathrm{~nm}) \\
\text { Poly dispersity index }\end{array}$ & Mobility & $-2.06\left(\mathrm{~cm}^{2} / \mathrm{Vs}\right)$ \\
0.33 & & \\
Diffusion Constant & Conductivity & $1.68(\mathrm{mS} / \mathrm{cm})$ \\
$\begin{array}{l}1.11\left(\mathrm{~cm}^{2} / \mathrm{sec}\right) \\
\text { Refractive index }\end{array}$ & Refractiveindex & 1.33 \\
1.33 & & \\
Viscosity & Viscosity & $0.89(\mathrm{cP})$ \\
$0.88(\mathrm{cP})$ & & \\
\hline
\end{tabular}

Stability studies conducted for the selected formulation (CPN6) from prepared nanospheres were stored in the high density polyethylene (HDPE) containers, tightly closed and kept at $45 \pm 2{ }^{\circ} \mathrm{C}$ and $75 \pm$
$5 \%$ relative humidity for 90 days. The samples were characterized for particle size, zeta potential, drug content estimation. Stability studies results have been reported in Table 4. There were no significant changes found in these tested parameters after the stability study period.

\section{Conclusion}

From the results, it was concluded that the prepared nanospheres formulation CPN 3 and CPN 6 showed sustained release for a long period of $24 \mathrm{hrs}$ with $95.32 \pm$ $0.45 \%$ and $94.28 \pm 0.20 \%$ releases, respectively. The release mechanisms for all the formulations followed by non-fickian diffusion mechanism because all the prepared formulations are having diffusion exponent value (n) more than 0.45 and less than 1 . The optimized nanosphere formulation CPN 6 could be employed to minimize the adverse effects of capecitabine.

\section{Acknowledgements}

The authors acknowledge Nishka Laboratories, Hyderabad, India for providing facilities to evaluate the formulations.

\section{References}

Bernardvan, E.G. and Patrick A. 2008. Top-down productionof drugs nanocrystals: Nanosuspension stabilization, miniaturization and transformation into solid products. International Journal of Pharmaceutics, 364, 64-75.

Dan L., Gary C. and Ronald B.. 2008. A Phase I Study of EKB569 in Combination with Capecitabine in Patients with Advanced Colorectal Cancer. Clin Cancer Res, 14, 5602.

Donbrow, M. and Samuelov Y. 1980. Zero order drug delivery from double-layered porous films: Release rate profiles from ethyl cellulose, hydroxypropylcellulose and polyethylene glycol mixtures. J. Pharm. Pharmacol, 32, 463-470.

Erem Bilensoya, Cansarisozena, Gunes. 2009. Intravesical cationic nanoparticles of chitosan and polycaprolactone for the delivery of Mitomycin $\mathrm{C}$ to bladder tumors. Intl. J. Pharm. 371, 170-176.

Higuchi, T. 1961. Rate of release of medicaments from ointment bases containing drugs in suspension. J. Pharm. Sci, 50, 874-875

Holunwong and Reina B. 2007. Chemotherapy with anticancer drugs encapsulated in solid lipid nanoparticles. Advanced Drug Delivery Reviews, 59, 491-504. 
Jasonpark, M.S. Peter. M. and Fong, Jinhlu, Kerry. 2009. PEGylated PLGA nanoparticles for the improved delivery of doxorubicin Nanomedicine. Nanotechnology, Biol. Med. 5, 410-418.

Lee, M., Cho, Y. and Chung, H. 2006. Sizecontrol of selfassembled nanoparticles by an emulsion/solvent evaporation method. Colloid Polym. Sci, 284, 506-512.

Leroux, J.C., Allemann. E. and Doelker. E. 1995. New approach for the preparation of nanoparticles by an emulsification diffusion method. Eur. J. Pharm. 41, 14-18.

Lifeng, Q.I., Jiang, X. and Yang, M. 2005. Cytotoxic activities of chitosan nanoparticles and copper-loaded nanoparticles. Bioorganic Med. Chem, 15, 1397-1399.

Mochizuki, M. and Hirami. M.1997. Structural effects on biodegradation of aliphatic polyesters. Polym. Adv. Technol. 8, 203.

$\mathrm{Mu}$, L. and Feng, S.S. 2003. A novel controlled release formulation for the anticancer drug paclitaxel (Taxol): PLGA nanoparticles containing vitamin E TPGS. J Control Rel. 86, 33-40.

Nazneen D., Jaya P. and S. Pujapanwarhazari. 2012. Preparation and biological evaluation of paclitaxel loaded biodegradable PCL/PEG nanoparticles for the treatment of human neuroendocrine pancreatic tumor in mice. Hell. J. Nucl. Med. 15, 9-15.
Quintanar, G.D., Ganem, Q.A., Allemann, E. and Fessi, H. 1998. Influence of the stabilizer coating layer on the purification and freeze drying of poly (DL-lactic acid) nanoparticles prepared by emulsification-diffusion technique. $J$. Microencapsulation. 15, 107-119.

Rahulnair, Arun Kumar, K.S. and Vishnupriya, K.V. 2011. preparation and characterization of Rizatriptan loaded solid lipid nanoparticles. J.Biomed Sci And Res. 3, 392-396.

Ritger, P.L. and Peppas, N.A. 1987. A simple equation for description of solute release II. Fickian and anomalous release from swellable devices. J. Control. Rel. 5, 37-42.

Sandri, 2010. Chitosan-associated SLN: in vitro and ex vivo Characterization of Cyclosporine A loaded ophthalmic systems. J. Microencapsul. 27, 735-746.

Wong, H.L., Rauth, A.M. and Bendayan, R.A. 2007. New polymer-lipid hybrid nanoparticle system increases cytotoxicity of doxorubicin against multidrug-resistant human breast cancer cells. Pharm Res. 23, 1574-1585.

Zhang, C., Ping, Q. and Shen, J. 2003. Synthesis and characterization of water soluble o-succinyl-chitosan. European Polymer J. 39, 1629-1634. 